\title{
Cerebral Hydatitosis: About 12 Observations at the University Hospital Center of Conakry
}

\author{
Fodé Abass Cisse $^{1,2}$, Foksouna Sakadi ${ }^{2, ~ *, ~ A m i n a ~ S a k h o ~}{ }^{3}$, Naby Camara ${ }^{1}$, \\ Barry Souleymane Djigué ${ }^{1,2}$, Arcel Steven Nitcheu Woga ${ }^{1}$, Nana Rahamatou Aminou Tassiou ${ }^{1}$, \\ Baldé Amadou Talibé ${ }^{1}$ Bi Krah Jean Bedel Ballo ${ }^{1}$, Mohamed Lamine Touré ${ }^{1}$, \\ Ibrahima Sory Souaré ${ }^{2,4}$, Amara Cisse ${ }^{1,2}$ \\ ${ }^{1}$ Neurology Department, Ignance Deen Teaching Hospital, Conakry, Guinea \\ ${ }^{2}$ Faculty of Medicine Pharmacy and Odontostomatology, University Gamal Abdel Nasser, Conakry, Guinea \\ ${ }^{3}$ Radiology Departement, Ignance Deen Teaching Hospital, Conakry, Guinea \\ ${ }^{4}$ Neurosurgery Department, Sino-guinean Hospital, Conakry, Guinea \\ Email address: \\ abass3002@yahoo.fr (F. A. Cissé), aminatasakho91@gmail.com (A. Sakho), nabylcp@yahoo.fr (N. Camara), \\ djigueb@yahoo.fr (B. S. Djigué), nitus25bbc@gmail.com (A. S. N. Woga), rah_tass@yahoo.fr (N. R. A. Tassiou), \\ baldeamadoutalib@yahoo.fr (B. A. Talibé), bedelbikrah@gmail.com (B. K. J. B. Ballo), touremed80.mlt@gmail.com (M. L Touré), \\ souareis@yahoo.fr (I. S. Souare), amaracisse69@yahoo.fr (A. Cissé), fokasaka@gmail.com (F. Sakadi) \\ ${ }^{*}$ Corresponding author
}

\section{To cite this article:}

Fodé Abass Cisse, Foksouna Sakadi, Amina Sakho, Naby Camara, Barry Souleymane Djigué, Arcel Steven Nitcheu Woga, Nana Rahamatou Aminou Tassiou, Baldé Amadou Talibé, Bi Krah Jean Bedel Ballo, Mohamed Lamine Touré, Ibrahima Sory Souaré, Amara Cisse. Cerebral Hydatitosis: About 12 Observations at the University Hospital Center of Conakry. Clinical Neurology and Neuroscience.

Vol. 2, No. 2, 2018, pp. 41-45. doi: 10.11648/j.cnn.20180202.14

Received: May 28, 2018; Accepted: July 3, 2018; Published: August 1, 2018

\begin{abstract}
Cosmopolitan disease, hydatidosis is caused by the hydatid larvae of a tapeworm of Echinococcus granulosus canes developing in the liver, lungs, heart and central nervous system. The biological certainty of brain damage in West Africa is difficult to confirm because of the supposed rarity of this affection and the difficulties of accessibility to MRI and CT radiological data suggestive of the disease. We retrospectively analyzed the file of 268 patients hospitalized in the Neurology Department between 2010 and 2016 for the management of encephalic syndrome with cystic neuroradiological cerebral lesions. Biological and neuroradiological evidence of hydatidosis was reported in 12 patients (4, 47\%). An encephalic and infectious syndrome: headache, nausea and vomiting, fever, disorders of consciousness, sensitivo-motor deficit with hemiparesis type, cerebellar syndrome and sometimes visual disorders, expression of intracranial hypertension was found in patients. These elements of intracranial hypertension objectified in most patients, were associated in 3 cases with liver disorders. The biological data haemagglutination, Eliza, moderate eosinophilia, radiological CT / MRI and the demonstration of scolex during percutaneous aspirations ( 2 cases) and on operative specimens were the diagnostic confirmation beam.
\end{abstract}

Keywords: Echinococcus Granulosus, Cerebral Hydatidosis, Albendazole, Conakry, Guinea

\section{Introduction}

The frequency of cerebral hydatidosis is variable in the literature and is around 0.5 to $4 \%[1,2,3]$ among other intracranial processes, especially in endemic countries: Australia, New Zealand, Mediterranean region, Argentina, Chile and some countries of Eastern Europe and Asia. In sub-
Saharan region of Africa, there is an underreporting of hydatidosis apart from a few series reported in Niger, Mali, Mauritania, Chad, Tanzania, Morocco, and especially in Kenya where the prevalence would reach $6.6 \%$ [3, 4].

Ubiquitous affection today, the hydatidosis is due to the development of the larvae of a tapeworm called Echinococcus granulosus that contaminates the man by the ingestion of food soiled by the dog excrement, this one thus 
becoming an intermediate host like the sheep.

This pathology causes hepatic, pulmonary and cardiac complications, of which cerebral localizations represent 1 to $2 \%$ of hydatid cysts in the human body $[1,2,5]$. In tropical environments, the difficulties of biological diagnosis and inaccessibility to neuroimaging lead us to report only isolated cases of truly documented hydatidosis [3].

We report the retrospective study of cerebral hydatidosis recorded in Guinea with the aim of reevaluation from the clinical and paraclinical point of view. The interest of this work resides in the fact that these observations illustrate the cerebral hydatidosis and the diagnosis difficulty that it involves in tropical environment, with the other lesions of cystic nature like abscess of the brain, leptomeninigated arachnoid cyst, porencephalic cavity and especially cystic astrocytoma and neurocysticercosis.

\section{Patients and Methods}

The 12 patients were hospitalized in the neurology department of the University Hospital Center of Conakry, the only establishment of the country for the care of patients suffering from chronic neurological affection.

All patients received a systematic clinical examination with medical-surgical antecedents and notification of all movements and trips to the Mediterranean and some African countries recognized as high endemicity as Kenya, Tanzania, Morocco.

All patients received a complete, central and peripheral neurological examination by a neurologist.

Nutritional status was also assessed by determining the Body Mass Index (BMI) of the World Health Organization (WHO); weight (in $\mathrm{kg}$ ); height ( $\mathrm{m} 2$ ) incidence of DETSKY [6] and albumin dosage. The biological assessment was performed in all patients: NFS, VS, CRP, fasting blood glucose, serum calcium, serum iron, ASAT, ALT, gamma-GT, free and conjugated bilirubin, ammonia, amylase, lipase, urea, creatinine, ionogram, proteinuria 24 hours.

All patients also have benefited from ECG, echocardiography, thoracic and cerebral CT scan. Brain MRI was performed in 3 patients. Hydatid serology by indirect haemagglutination with threshold of positivity of $1 / 320$ in Elisa. Casoni intradermal reaction was not performed.

The 9 patients have been operated and the 3 others by percutaneous aspiration had an anatomopathological examination and the microscopy of the fluid.

Electroencephalographic examination was performed in all patients. The plots were classified into 3 types.

Type I:

1. EEG with dominance of alpha rhythm, parieto-occipital topography whose amplitude is greater than $40 \mathrm{Mv}$ without pathological rhythms.

2. EEG without dominance proper with existence of irregular alpha rhythms without the presence of pathological waves.

3. EEG with dominance of beta rhythm of topography especially before Rolando's fissure and whose amplitude reaches $20 \mathrm{Mv}$.

Type II:

1. EEG without dominance well said but with the appearance of theta rhythms of 4 to $12 \mathrm{~Hz}$ temporoparietal topography of small amplitudes 30 to $40 \mathrm{Mv}$ isolated sometimes diffuse or grouped under paroxysmal flushes.

Type III:

1. EEG with theta or delta rhythms with abnormal spikewave patterns;

2. EEG with prevalence of pathological theta and delta rhythms without precise localizations in the form of general asymmetric delta-theta dysrhythmias.

The therapeutic approach consisted of a surgical treatment by one-piece resection of the cyst while avoiding an intraoperative rupture of the cyst.

In 3 cases, due to no-acceptance of patients for surgery, a simple puncture of the cyst was performed.

All patients were bacteriocidal with albendazole at a dose of $10-15 \mathrm{mg} / \mathrm{kg} /$ day in 2 doses for 30 days with evaluation of efficacy in 8 months.

\section{Results}

The analysis of the results of this study focused on the clinical (Table I), biological, neuroradiological, electrophysiological and therapeutic data. The 12 cases were collected during the study period.

The means age is 22.6 years old (extreme 16 and 48 years); the delay between the onset of clinical manifestations and the consultation or hospitalization was only precise in 5 patients between 6 months and 10 months; the other patients first underwent a traditional medicine itinerary for the management of headaches; nausea and neurological deficit at home.

The antecedents were search in all cases: 6 patients stayed during the course of their studies in North Africa and 2 tradesmen. In 4 cases, no notion of travel to endemic areas was notified, but all of them coming from the average Guinea, the country's favourite breeding zone: sheep, goats. These 4 cases are considered as autochthonous (Figure 1).

The evaluation of nutritional status according to BMI demonstrates the existence of malnutrition associated with hydatidosis for seven patients. This result was confirmed by the DETSKY index, which is based on clinical and subjective approach that has made it possible to classify our 7 patients in groups $\mathrm{B}$ and $\mathrm{C}$ corresponding to undernourished patients. The clinical signs at the start and state stage are summarized in Table 1. 
Table 1. Clinical signs at onset and advance stage of disease.

\begin{tabular}{lc}
\hline Clinical signs at the beginning phase & Frequency (\%) \\
\hline hyperthermia (fever $>38^{\circ} \mathrm{C}$ ) & $5(41,69)$ \\
Headaches (progressive aggravation resistant to analgesics sometimes associated with nausea or vomiting) & $11(91,7)$ \\
Dizziness (vertiginous paroxysms per moment) & $2(16,7)$ \\
Cervico-occipital pains (diffuse irradiation aggravated by neck movements) & $2(16,7)$ \\
Digestive disorders (contemporary vomiting with cephalalgic attacks, fatigability and irritability) & $8(66,7)$ \\
Clinical signs at the state phase & $7(58,3)$ \\
Disorders of consciousness (confusional syndrome, bradypsychia, loss of initiative and ideological alteration) & $10(83,3)$ \\
Epileptic seizures (partial, generalized tonic-clonic, complexes temporalis, state of evil) & $12(100)$ \\
Neuroradiological signs in focus (right or left hemiparesis, aphasia, homonymous lateral hemianopia or quadranopsia) & \\
\hline
\end{tabular}

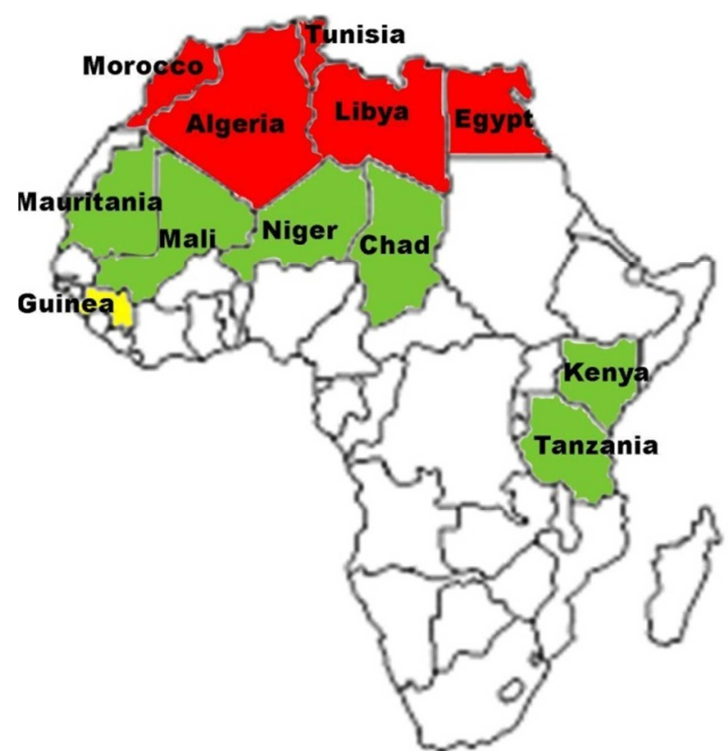

Figure 1. Neuroeoidemiology.

Green: Country where few cases have been identified.

Red: Countries at high risk of endemic.

Yellow: Guinea.

In two patients, the clinical examination noted abdominal pain, right hypochondrium gravity, and moderate hepatomegaly, responding to GHARDI stage II and ultrasonography revealed liver cysts.

The headaches associated with epileptic seizures with focal neurological signs constitute the essence of the call and state semiology of cerebral hydatidosis.

In 2 cases, status epilepticus with generalized tonic-clonic seizures was the revealing factor of the condition. In 7 cases, confusion syndrome was identified in a nutrition context in patients classified in group B and C of DETSKY.

\subsection{Biological Data}

Biologically, abnormalities of the blood count were noted in most patients with an average hemoglobin level of $8.62 \pm$ $2.3 \mathrm{~g} / \mathrm{dl}$; Eosinophilia was reported in 4 patients (33\%). In 2 cases, the ASAT level was between 210 and $230 \mathrm{IU} / \mathrm{ml}$ and the globulins were increased, the serum albumin levels were decreased $(22 \pm 5.5 \mathrm{~g} / \mathrm{l})$. In the other groups, abnormalities in blood count, ionogram, and liver and kidney status were poorly disturbed.

\subsection{Electrophysiology Data}

Plots of type II (2 cases) and III (9 cases), classified pathological in the form of generalized Theta and Delta dysrhythmias especially in which had been noted the comitiales seizures and the disorders of the conscience. Type I, considered normal, was only observed in one patient.

\subsection{Neuroradiological Data}

In nine patients, the cerebral CT scan showed a cyst content with a density close to that of water, with the injection of the contrast medium a thin and well-defined spherical shell (Figures 2, 3, 4). Sometimes with perilesional mass effect (Figure 5).

In 3 patients, the MRI revealed a round spherical formation, well limited in hyposignal $\mathrm{T} 1$ and hypersignal T2 with a thin pavis.

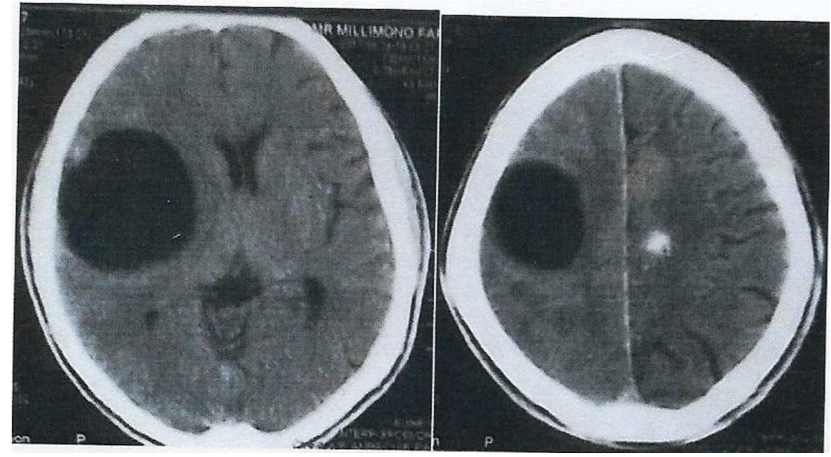

Figure 2. CT scan without injection in axial section showing large right hemispherical non-calcified cysts and small left hemispherical calcified cyst.

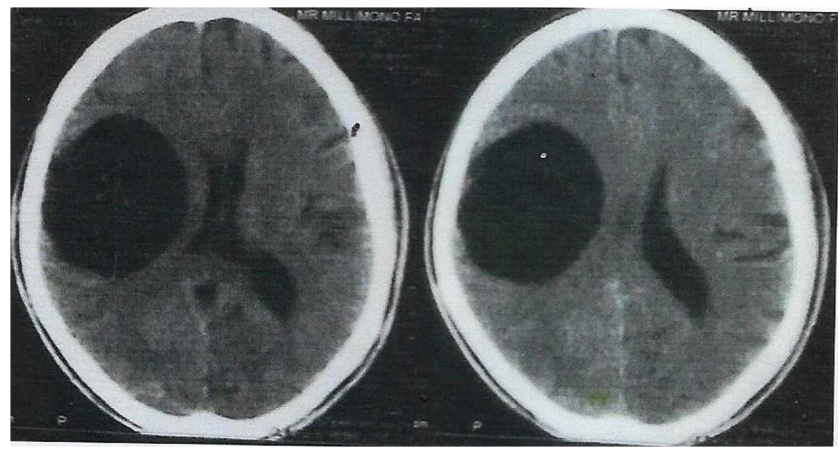

Figure 3. CT scan without injection in axial section showing large right hemispherical cysts 


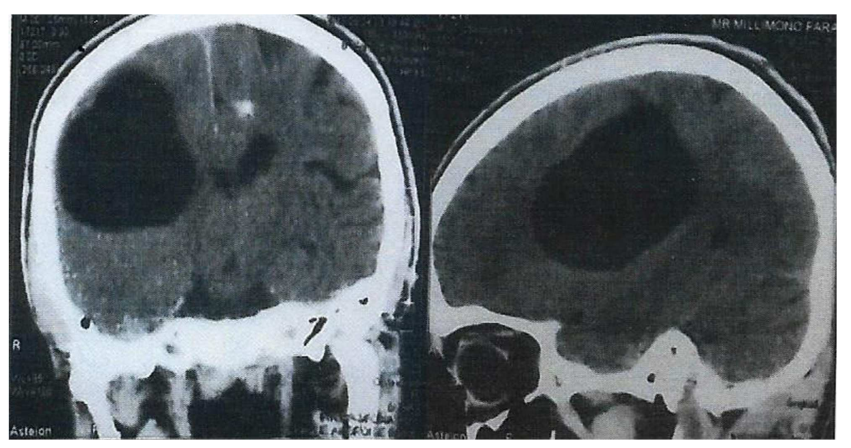

Figure 4. Cerebral CT without injection in sagittal and frontal section showing a large cyst with cerebral edema

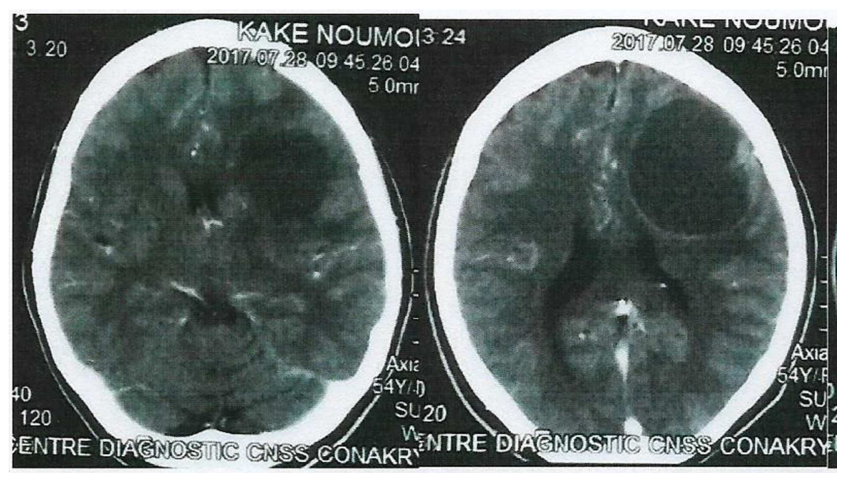

Figure 5. CT scan with axial section injection showing a large cerebral cyst with a large left hemisphere mass effect.

\subsection{Therapeutic Data}

The decompressive surgery associated with the institution of a medical treatment at the dose of 10 to $15 \mathrm{mg} / \mathrm{kg}$ weight in 2 taken for 30 days allowed an improvement in 7 cases after a first evaluation of 6 months, 2 cases of death at undernourished patients and 3 cases lost to follow-up.

In tropical environments, clinical, neuroradiological and neuropathological examination has eliminated in all cases, brain abscesses common in our region, other cystic lesions including leptomeningeal arachnoid cyst, squamous, cystic astrocytoma.

\section{Discussion}

This study is, to our knowledge, one of the most important series of documented brain hydatidosis in West Africa. Twelve cases of neurological manifestations of hydatidosis were collected in this retrospective study over a period of 6 years. These are all suspected cases or some whose confirmation diagnosis has been notified to the University Hospital of Conakry.

Work on hydatidosis shows that in countries with recognized endemicity, hepatic localization accounts for $50 \%$ and pulmonary $36 \%$, whereas cerebral sites are only $2 \%$ [2]. The data in the literature $[3,5,6]$ show that cysts are mostly found in the supratentorial and subcortical hemispherical zones mainly involving the vascularization zones of the middle cerebral artery, as demonstrated by our series.

However LAKHDAR and al. [7, 8] reported series of interesting posterior cerebral form. Most authors note that cerebral localizations are in 10 to $15 \%$ associated with other visceral localizations especially hepatic and pulmonary. In this study we only noted this association in two patients with liver cysts.

It has been established since old publications that the major endemic foci are the Mediterranean Basin, South America, Australia, New Zealand, parts of Eastern Europe where extensive livestock sheep and in some cases with coexistence of dogs $[1,2]$.

In West Africa, there is a real underreporting of this pathology due to under-medicalization, inaccessibility to neuroradiological diagnosis and weak biological explorations. Today the mixing of populations with North Africa and uncontrolled migrations will make this pathology a public health problem in the future.

Classically, cerebral hydatidosis is found in children and adults, $[3,5]$, a result that we find in this study.

In West Africa, particularly in Guinea, cerebral hydatidosis is often associated with other factors: vitamin deficiency, eating disorders as demonstrated by the nutritional evaluation of some of our patients by the determination of the Body Mass Index (BMI) of WHO and DETSKY.

In general, the clinical pictures of cerebral hydatidosis observed in this study do not differ from those described in the literature with severe entities because of the combination of undernutrition and consultation delays.

The clinical forms observed here correspond to the known clinical and neuroradiological features of CT / MRI [9]. Nonetheless, there are serious forms of intracranial hypertension, confusional syndrome and status epilepticus related to the importance of consultation delays and patients' journey through traditional medicine.

The brain forms of cerebral hydatidosis associated with the hepatic one often pose diagnostic problems because in Africa hepatic encephalopathies are multifactorial, hence the importance of an exhaustive liver assessment in search of other infectious, bilharzeal causes.

On the paraclinical level, the CT scan was very informative by highlighting a very suggestive cyst appearance: cyst content with a density close to water, with infection of contrast material revealing a thin splenic shell and limited site, rarely or not at all in contrast to MRI [1].

Most authors [2, 5, 10] believe that magnetic resonance imaging, by showing a low signal in $\mathrm{T} 1$ and high signal in $\mathrm{T} 2$, is especially indicated in the demonstration of the multiple hydatid cysts and the lesions of the neighboring structures helping thus the surgical act.

The search for anti-echinococcosis antibodies may be a factor entering the diagnosis beam with significant haemagglutination at 1/320; however, some authors believe that an isolated and unbroken intracranial hydatid cyst is negative $(30 \%)$.

Therapeutically, surgical excision of the cyst represents the route of intake in recharge, associated with albendazole to avoid recovery of the lesion $[11,12]$. An assessment is required at 9 months, 12 months and 2 years. 


\section{Conclusion}

Cerebral hydatidosis, which has not been widely reported in West Africa in recent years, is becoming an increasingly frequent condition, due to the existence of indigenous forms of migration and population movements to recognized areas of endemicity.

Thus the diagnosis of hydatid cyst is evoked in front of any syndrome of intracranial hypertension with the institution of explorations neuroradiological (CT) and biological (haemagglutant, ELIZA).

Management is based on surgery, however early and wellmanaged medical treatment improves the patient's clinical condition.

\section{References}

[1] I. Lotfinia; Central Nervous System Hydatid Disease; SMGroup, 2017 (p1-26), www.smgebooks.com

[2] J. Maraby-Salgado, J. Mo-Carrascal, J. Aquino-Matus, W. G Calderon-Miranda, A. Agrawal, A. F et al.: Brain hydatidosis: review of the literature; Romanian Neurosurgery, 2017 (p1-8), Volume XXXI, Number 3; DOI: 10. 1515/romneu-2017-0061

[3] A. Pierre: Hydatidose ou kyste hydatique Actualites 2013. Medecine Tropicale www, edecinetropicale.com.

[4] L. Benantar, K. Aniba, M. Laghmari, M. Lmejjati, H. Ghannane, S. Ait Benali; La prise en charge de l'hydatidose du système nerveux central; Neurochirurgie 63 (2017) 31-52, O50; http://dx.doi.org/10.1016/j.neuchi.2016.11.052.
[5] A. El Saqui, M. Aggouri, M. Benzagmout, K. Chakour, M. El Faiz Chaoui; Kystes hydatiques cérébraux de l'enfant: à propos de 15 cas; Pan African Medical Journal. 2017; 26:205 doi: 10.11604/pamj. 2017. 26. 205. 8398

[6] DETSKY A. S, Mc Laughin JR, Baker JP et al. Whatis subjective global assessment of nutritional STATUS. J. PEN J. Parenter Enteral Nutrition 11 (1) 8-13.

[7] LAKhdar F. ARKha Y, Bougrine M, DERRAZ S, EL Ouhabi A, EL KHAMLICHI A. Kyste hydatique intre et extracranien de la fosse cerebrale posterieure (à propos d'un cas) Neurochirurgie 2010, 56, 391-394.

[8] Bakhsh A, KMA S, Taraif S. Primary hydatid cyst of pineal region of brain: a case report from Saudi Arabia. Asian J Neurosurg. 2017; 12 (2):314 - 7.

[9] TUZUN M, ALTINORSN, ARDA IS, Heki, oglu B cerebral hydatic disease CT scan MRI Finding clin Imaging 2002, 26, 353-357.

[10] M. D Aydin, N. C Karaavci, M. E Akyuz, M. H Sahin, M. Zeynal, A. Kanat et al.; A New Technique in Surgical Management of the Giant Cerebral Hydatid Cysts; Journal of Craniofacial Surgery; Vol. 00, N. 00, 2018 (p1-5); 10. 1097/SCS. 0000000000004236

[11] S. Chen, N. Li, F. Yang, J. Wu, Y. Hu, S. Yu; Medical treatment of an unusual cerebral hydatid disease; BMC Infectious Diseases; 2018 (p1-4); 10. 1186/s12879-017-29352 .

[12] Fattahi Masoom SH, Lari SM, Fattahi AS, Ahmadnia N, Rajabi M, NaderiKalat M. Albendazole therapy in human lung and liver hydatid cysts: a 13-year experience. Clin Respir J. 2017; https://doi.org/10.1111/crj.12630. 